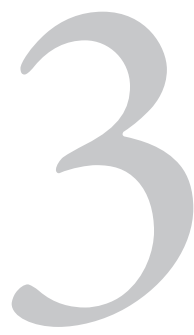

\title{
CONSTRUCCIÓN DE UN ÍNDICE PARA MEDIR LA TRANSPARENCIA MUNICIPAL: BUENOS AIRES, BAHÍA BLANCA Y LAS CAPITALES DE PROVINCIA DE ARGENTINA
}

\section{BUILDING AN INDEX TO MEASURE MUNICIPALITIES' TRANSPARENCY : BUENOS AIRES, BAHÍA BLANCA AND THE CAPITAL STATES IN ARGENTINA}

\author{
Franco Ciucci ${ }^{2}$ \\ Lucía Díaz ${ }^{3}$ \\ María Verónica Alderete ${ }^{4}$ \\ Sebastián Linares ${ }^{5}$
}

Recibido: 03/05/2019

Aceptado: 02/07/2019

\section{RE S U M E N}

Este trabajo estudia el principio de transparencia del paradigma de gobierno abierto. Con el objetivo de cuantificar la transparencia en el nivel municipal, se construye un índice de transparencia activa presupuestaria y transparencia pasiva. Éste se calcula tomando en consideración el aporte teórico de Darbishire (2010) y sobre una muestra de 25 ciudades de Argentina (capitales de provincia junto a Ciudad de Buenos Aires- CABA- y Bahía Blanca). Se exploran los sitios web municipales para determinar la presencia o no de información presupuestaria y de gasto público, así como

1. El presente trabajo fue realizado en el marco del proyecto Unidad Ejecutora IIESS (PUE: 22920160100069C0): Inclusión social sostenible: Innovaciones y políticas públicas en perspectiva regional (financiado por CONICET, Argentina).

2. Departamento de Economía, Universidad Nacional del Sur. Bahia Blanca, Argentina.

Contacto: francociucci@hotmail.com

3. Instituto de Investigaciones Económicas y Sociales del Sur, Universidad Nacional del Sur-CONICET. Bahía Blanca, Argentina. Contacto: Iucia.diaz@uns.edu.ar

4. Instituto de Investigaciones Económicas y Sociales del Sur, Universidad Nacional del Sur-CONICET. Bahía Blanca, Argentina Contacto: mvalderete@iiess-conicet.gob.ar

5. Instituto de Investigaciones Económicas y Sociales del Sur, Universidad Nacional del Sur-CONICET. Bahía Blanca, Argentina Contacto: slinares@usal.es 
la existencia de un sistema centralizado de gestión de las solicitudes de información y la publicación de las respuestas para su aprovechamiento público. Según los resultados obtenidos, los municipios más transparentes son Córdoba, CABA, Bahía Blanca y Ushuaia. Posteriormente, se analiza el nivel de transparencia en relación al tamaño y nivel de ingresos de los municipios, hallándose cierta evidencia de una relación positiva.

PA L A B A S C L A VE S : gobierno abierto, transparencia, TIC, índice sintético, municipios.

\section{A B S T R A C T}

This paper studies the transparency principle as one of the axis of the open government paradigm. To quantify transparency at the municipal level, an index of budget active transparency and passive transparency is built It is calculated by using several theoretical baselines of Darbishire (2010), which is applied to a sample of 25 cities from Argentina (the capital states together with Buenos Aires and Bahia Blanca). Officials websites of these municipalities are explored to determine the presence or not of budget and public expenditure items and presence of an e-petitioning system of public information with their respective responses for public use. Based on the results obtained, the most transparent capital states in Argentina are Cordoba, Buenos Aires (CABA), Bahia Blanca and Ushuaia. Afterwards, the level of transparency is analysed in relation to the municipalities' size and income levels, finding some evidence of a positive relationship.

K E Y W O R D S : open government, transparency, ICT, synthetic index, municipalities.

\section{N T R O D U C C I Ó N}

Todas las estructuras institucionales generan datos de manera constante, pero no todos los datos se publican ni resultan asequibles para la ciudadanía. Todos los gobiernos y estructuras institucionales tienen incentivos para ocultar datos que les perjudican, y para publicar datos que los benefician electoralmente. Sin embargo, los datos producidos por el gobierno, si bien están en posesión de las autoridades oficiales, pertenecen al conjunto de los ciudadanos. Si la libertad de expresión es un derecho nuclear de la democracia, el derecho a acceder a la información pública es su correlato directo, ya que una democracia que tenga un compromiso con la evaluación de políticas basadas en evidencias necesita garantizar el acceso ciudadano 
a los datos que genera el Estado. Sin ese compromiso, los ciudadanos podrán ejercer su libertad de expresión, pero no podrán ejercer una crítica al gobierno fundada en evidencias.

En el ámbito político, el acceso y la publicación de esos datos han sido y son causa de numerosas discusiones y regulaciones. Por un lado, una buena parte de la información que se recolecta hace referencia a personas ajenas a la administración pública, personas que tienen un interés legítimo de que sus datos personales sean protegidos en resguardo de su intimidad. Por otro lado, muchos de estos datos, en su carácter agregado (es decir, sin información personal), así como otros datos referidos a la actividad del Estado, son de interés público por distintas razones para al menos una parte de la población, y deberían ser de acceso público. Muchas democracias avanzadas del mundo comenzaron dando respuesta a este derecho ciudadano sancionando leyes regulando el acceso ciudadano a la información pública (Freedom of Information-FOI). Los avances en transparencia son el resultado de un proceso histórico, a través del cual fueron surgiendo demandas concretas ciudadanas por una mayor transparencia y rendición de cuentas, que chocaron contra una cultura estatal de opacidad y secretismo. Es en el fragor de esta conflictividad que las leyes de acceso a la información fueron adquiriendo forma (Krastev, 2004; Pereyra, 2013, Lascoumes, 1999). Los cambios tecnológicos de la revolución digital han transformado los incentivos y los costos de la transparencia, coadyuvando a una mayor presión por dar a conocer datos relevantes de manera oficiosa En una segunda oleada de reformas, se consideró necesario ir más allá y publicar, de oficio, datos que son de relevancia pública. Surgieron así las leyes de transparencia activa, a través de las cuales se obliga a las instituciones oficiales a publicar determinados datos e información (Noveck, 2017). Tenemos entonces dos dimensiones relevantes de la transparencia y el acceso ciudadano a la información pública: la transparencia activa (que vuelve operativo el deber del Estado de proveer información) y la transparencia pasiva (que vuelve operativo el derecho de acceso a la información) (Noveck, 2017)”. La pregunta que surge entonces es: ¿los ciudadanos efectivamente tienen derecho a acceder a la información que está en manos del gobierno (sea local o central)? En materia de transparencia activa: ¿Es posible calcular el nivel de apertura efectivo de tales datos por los gobiernos y comparar entre ellos?

En este trabajo aspiramos a trazar una radiografía de la transparencia presupuestaria activa y de la transparencia pasiva de los municipios de las ciudades capitales de las Provincias Argentinas, más la ciudad de Bahía Blanca (que no es capital) y la Ciudad de Buenos Aires (que no encuadra exactamente dentro del nivel municipal). Estas dos últimas se agregaron sólo a los efectos de introducir dos ciudades que cuentan con una reputa- 
ción prestigiosa de apertura de datos, para ver si se trata de una reputación fundada en evidencias y para verificar en qué medida el resto de capitales de provincia se posiciona en relación con ellas.

¿Por qué escogimos el nivel municipal y por qué las capitales? Primero, porque son escasos los estudios empíricos de transparencia municipal, y allí donde es posible encontrar estudios de casos, resulta difícil su comparación con otras realidades ya que los parámetros que se utilizan suelen ser idiosincráticos. Para comparar hacen falta parámetros comunes que se apliquen a la muestra seleccionada de manera uniforme, y eso es precisamente el objetivo de este estudio. Las capitales de Provincia fueron seleccionadas por economía de trabajo: sencillamente, no disponemos de los recursos para encarar una comparación global de todos los municipios argentinos. Sin embargo, nuestra hipótesis de trabajo es la de que las capitales tienen una relevancia política institucional que debería de facilitar el avance hacia una mayor transparencia, o al menos la condición de capital no debería ser un obstáculo para una mayor apertura.

Este trabajo se estructura de la siguiente manera. La primera sección presenta el marco teórico y conceptual del trabajo, haciendo énfasis en la noción de transparencia activa y pasiva. La segunda sección ofrece una revisión de los índices de transparencia existentes en la región de América Latina, y en particular en Argentina. En tercer lugar, se describe la metodología utilizada para la confección del índice. Luego, se analizan los resultados obtenidos y por última, se brindan algunas consideraciones finales.

\section{MAR C O TE ÓR I C O}

La cuestión del acceso a la información pública se plantea normalmente como un derecho extenso que no merece lugar a reparos. Sin embargo, es objeto de los mayores conflictos institucionales porque usualmente los funcionarios no desean que los datos que se generan estén en manos de todos. Ello se debe a que el control de la información implica poder y al compartir los datos se diluye esa potestad. Además, existe el temor -a veces fundadode que los datos publicados puedan ser utilizados en contra de los intereses del gobierno o de sus representantes. Por tal razón, la regulación estricta acerca de cuándo procede dar acceso a la información es una forma útil para evitar numerosos conflictos sociales.

Para comprender los fundamentos de la apertura de los datos de gobierno, corresponde distinguir según se enfoque a la cuestión como un derecho de 
los ciudadanos o como una herramienta para mejorar la dinámica social (Ubaldi, 2013). En el primer caso, la razón de ser del acceso a la información pública es de tipo filosófico y se justifica en que los gobernantes son mandatarios que trabajan en representación de los intereses de la población. Por ende, la apertura de la información es un buen mecanismo para una rendición de cuentas eficaz y continua. En el segundo caso, a menudo se destacan las ventajas que otorga a la ciudadanía el hecho de poder acceder a los datos del gobierno, por ejemplo, acerca de los servicios que ofrece la ciudad, la provincia o la Nación. Además, a las empresas les puede ser útil para reducir costos de investigación y sondeo previo a realizar una inversión. Lo que en economía se conocen como costos de transacción (Coase,1937; Williamson, 1981). En este sentido, los datos abiertos permiten reducir los costos de transacción. Ambas perspectivas son complementarias, pero la primera sentó las bases para la sanción de leyes regulatorias de la transparencia pasiva (FOI), y la segunda da fundamento a las leyes de transparencia activa.

En definitiva, hay razones teóricas y prácticas para que los datos del Estado se compartan con la ciudadanía. Surge así un nuevo servicio público que es la apertura de Datos Abiertos Gubernamentales que se brinda a través de un Portal de Datos Abiertos (aspecto técnico) y cuyo fin es promover su reutilización por parte de la sociedad, para fines de transparencia de la Administración Pública, y para promover el desarrollo social y económico (aspecto social y económico). Según la Secretaría de Modernización de la República Argentina, un dato abierto de origen público es aquel dato al que cualquier persona puede acceder, usar y compartir libremente. Sólo deben atribuirse y compartirse con la misma licencia con la que fueron publicados. Son, a su vez, datos públicos disponibilizados en un medio digital, bajo una licencia abierta y usando para ello un formato estándar abierto, si bien este último recaudo no siempre se respeta.

Se puede definir a la transparencia activa como la obligación de la administración pública de publicar y mantener actualizados ciertos ítems, datos o documentos que contienen información pública relevante. Esto es, aquellos elementos o datos considerados relevantes que va produciendo la Administración deberían ser visibles, localizables, accesibles y expresados con claridad. Tradicionalmente se utilizaban archivos documentales y repositorios, aunque la orientación moderna es la de suministrar la información on-line, en la web. La transparencia pasiva se puede caracterizar como un derecho de ciudadanía a recibir la información pública que solicite. Ello implica contar con canales adecuados para enviar la solicitud y un tratamiento y entrega de información particular para cada caso. El objetivo es que dicho 
procedimiento sea amigable, ágil y eficiente. Otra vez, la orientación moderna es que el procesamiento de la solicitud, su gestión y respuesta sea a on-line, en la web, y que cualquiera pueda ver el estado de la solicitud y cualquiera pueda acceder a la respuesta. Este último recaudo es crucial para determinar el compromiso de una administración con los datos abiertos. $\mathrm{Y}$ es que, si las respuestas a solicitudes particulares no se publican para que cualquiera pueda reutilizarlas, la información pública ya no es un bien público, sino un bien en red. Para poner un ejemplo: 5 personas conocen $X$ e $\mathrm{Y}$, pero sucede que $\mathrm{X}$ es de conocimiento público e $\mathrm{Y}$ es de conocimiento privado. Es decir, las personas saben que los demás saben $X$, pero ninguno sabe que los demás saben $Y$. Mientras que $X$ es un bien público, $Y$ es un bien en red. Pues bien, un sistema que vuelve públicamente disponible las respuestas particulares convierte a los datos en un bien público.

En este aspecto, Darbishire (2010) constituye un aporte para avanzar en la evaluación de la implementación de los principios de gobierno abierto por parte de los gobiernos, en particular respecto de las medidas de transparencia activa y pasiva. Al respecto, la autora se refiere al concepto de Transparencia Proactiva y menciona la necesidad de proveer de información al público necesaria para acceder a los servicios gubernamentales, que se han expandido ampliamente con el auge del gobierno electrónico. La autora ofrece un cuadro comparativo de casos con información sobre los estándares necesarios por parte de los gobiernos para alcanzar la transparencia. ¿Qué factores explican el grado de transparencia activa y pasiva? ¿Por qué algunos gobiernos exhiben una mayor transparencia y otros no? En la literatura empírica aún no tenemos respuestas generales con capacidad para "viajar" a una gran diversidad de contextos. Sí sabemos que las prácticas de transparencia y participación ciudadana propias del gobierno abierto están relacionadas con el tamaño de los gobiernos de referencia. Al respecto, Norris y Moon (2005) encontraron que, en el caso de los municipios norteamericanos, el tamaño medido por la cantidad de habitantes o población está estrechamente relacionado con la publicación de datos y la implementación de los servicios en línea Varios autores (Serrano-Cinca et al, 2009; Wilkinson y Cappel, 2005; Moon, 2002) indicaron que cuanto más grande es el municipio es más probable que el mismo adopte mayores prácticas de transparencia y gobierno electrónico comparado a los municipios pequeños. Por otro parte, cuanto mayor es la población mayor es la probabilidad de encontrar municipios con actividades participativas (Salvador et al., 2004; Criado, 2004; Blanco y Font, 2005; Brown y Schelin, 2005), otra dimensión -distinta a la de transparencia- que suele ir asociada a la noción general de gobierno abierto. Según parece, el tamaño de la ciudad importa para promover la transparencia (Albalate del Sol, 2013; Bearfield y Bowman, 2016). En las 
ciudades grandes la competencia política estimula la transparencia, mientras que en las ciudades pequeñas la escasez de recursos gubernamentales y el menor nivel de profesionalismo administrativo redunda en una menor transparencia.

En Argentina, el acceso a la información pública está regulado por la ley 27275 y recoge reglas aplicables tanto a la transparencia pasiva como activa. Con respecto a la primera, regula el procedimiento para la solicitud de información por parte de los ciudadanos, a la vez que determina los supuestos fácticos en los cuales procede y lo interpreta de la más amplia forma. Es así que el artículo $2^{\circ}$ establece que "el derecho de acceso a la información pública comprende la posibilidad de buscar, acceder, solicitar, recibir, copiar, analizar, reprocesar, reutilizar y redistribuir libremente la información" y se lo reconoce como un derecho fundamental independientemente de la existencia de un derecho subjetivo o interés legítimo, es decir toda persona humana puede solicitar información a los entes estatales (art. $3^{\circ}$ ). Con respecto al ámbito de aplicación de esta ley, el mismo se restringe a los tres poderes nacionales (Ejecutivo, Legislativo y Judicial) y a los entes descentralizados de la Nación. Es decir, no es directamente aplicable a las provincias ni a las municipalidades, sin embargo, tiene un valor ejemplificador que lleva que las instituciones subnacionales regulen la materia -en muchos casos- de forma muy similar a la legislación nacional. Las provincias, de hecho, pueden adherir a la ley, incorporando de esta manera su contenido a la normativa provincial ${ }^{6}$.

Un aspecto crucial de la ley son las excepciones al derecho a la información, las cuales se fundamentan en la necesidad de mantener ciertas materias ajenas al conocimiento público ${ }^{7}$. Por otro lado, lo que constituye una novedad, es que esta ley recoge la transparencia activa como una obligación de los funcionarios públicos. En primer lugar, establece que "deberán facilitar la búsqueda y el acceso a la información pública a través de su página oficial de la red informática, de una manera clara, estructurada y entendible

6. La Provincia de Buenos Aires, al momento de escribir este artículo, aún no ha adherido a la misma, con el argumento de que ya posee una normativa propia de transparencia (Ley 12.475/2000 y su Decreto reglamentario 2.549/04). Sin embargo, mientras que la ley nacional regula la transparencia activa, las normas provinciales sólo regulan la transparencia pasiva del gobierno provincial, y la Ley orgánica de Municipalidades tampoco establece criterios obligatorios de transparencia activa a ser publicados en la web. El resultado es que los Municipios de la Provincia de Buenos Aires tienen libertad para autorregularse en materia de transparencia pasiva y activa.

7. Las excepciones previstas son: información calificada como reservada por razones de política exterior; la protección de secretos industriales, comerciales o financieros; datos que afecten los derechos de un tercero; información cuya publicación pudiera afectar los resultados de un juicio o una investigación; información que pudiera comprometer la seguridad o vida de una persona y la información afectada por el secreto profesional (art. $8^{\circ}$ ). 
para los interesados" (art. 32). Además, se especifica cuáles son los datos que se deben hallar en los portales web de las instituciones obligadas. Entre la información que obligatoriamente debe encontrarse en las páginas $\left(\right.$ art. $\left.32^{\circ}\right)$, se destaca:

Un índice de la información pública que estuviese en su poder con el objeto de orientar a las personas en el ejercicio del derecho de acceso a la información pública, indicando, además, dónde y cómo deberá realizarse la solicitud;

Su estructura orgánica y funciones;

La nómina de autoridades y personal de la planta permanente $y$ transitoria $u$ otra modalidad de contratación, incluyendo consultores, pasantes y personal contratado en el marco de proyectos financiados por organismos multilaterales, detallando sus respectivas funciones y posición en el escalafón;

Las escalas salariales, incluyendo todos los componentes y subcomponentes del salario total, correspondientes a todas las categorías de empleados, funcionarios, consultores, pasantes y contratados;

El presupuesto asignado a cada área, programa o función, las modificaciones durante cada ejercicio anual y el estado de ejecución actualizado en forma trimestral hasta el último nivel de desagregación en que se procese;

El listado de las contrataciones públicas, licitaciones, concursos, obras públicas y adquisiciones de bienes y servicios, especificando objetivos, características, montos y proveedores, así como los socios y accionistas principales, de las sociedades o empresas proveedoras;

Los informes de auditorías o evaluaciones, internas o externas, realizadas previamente, durante o posteriormente, referidas al propio organismo, sus programas, proyectos y actividades;

Los permisos, concesiones y autorizaciones otorgados y sus titulares;

Los servicios que brinda el organismo directamente al público, incluyendo normas, cartas y protocolos de atención al cliente;

Todo mecanismo o procedimiento por medio del cual el público 
pueda presentar peticiones, acceder a la información o de alguna manera participar o incidir en la formulación de la política o el ejercicio de las facultades del sujeto obligado;

Un índice de trámites y procedimientos que se realicen ante el organismo, así como los requisitos y criterios de asignación para acceder a las prestaciones;

Mecanismos de presentación directa de solicitudes o denuncias a disposición del público en relación a acciones u omisiones del sujeto obligado;

Una guía que contenga información sobre sus sistemas de mantenimiento de documentos, los tipos y formas de información que obran en su poder y las categorías de información que publica;

La información que responda a los requerimientos de información pública realizados con mayor frecuencia; y

Las declaraciones juradas de aquellos sujetos obligados a presentarlas en sus ámbitos de acción.

Por último, la ley declara aplicables a la transparencia activa las excepciones generales a las obligaciones de transparencia pasiva, en particular lo concerniente a la protección de datos personales (art. $34^{\circ}$ ). En síntesis, esta regulación legal es una de las modernas y exigentes con los funcionarios públicos. Su ventaja principal reside en que no se limita a regular la transparencia pasiva, sino que incorpora -como es la tendencia a nivel mundialla exigencia de que sea la propia Administración la responsable de acercar la información a la ciudadanía. Ello es indispensable para garantizar una mejor participación ciudadana, una rendición de cuentas más eficaz y un aumento en la eficiencia de los servicios sociales que brinda el Estado.

\section{A ME D I C IÓN DE LA TRANSPARENCIA E N A M E R I C A L AT INA}

Desde hace más de dos décadas que los países de América Latina vienen trabajando en políticas de gobierno abierto, ya sea a partir de la implementación de convenciones anticorrupción, de los esfuerzos de modernización del Estado mediante la utilización de tecnologías y la descentralización de servicios públicos, o con la promulgación y entrada en vigencia de leyes de acceso a la información pública (Ramírez Alujas y Dassen, 2014) 
En los últimos años la región ha sido escenario de nuevas experiencias de gobierno abierto, evidenciándose en el hecho de que en la actualidad 16 países se encuentran adscritos a la Alianza por el Gobierno Abierto (AGA) ${ }^{8}$ (Hoffman et Al, 2013; Oszlak y Kaufman, 2014). Tales países han presentado e implementado planes de acción que contienen un número de compromisos concretos, mensurables, con fechas estimadas de implementación en el corto o mediano plazo, y la indicación de los organismos responsables de cumplirlos (Ramírez Alujas y Dassen, 2014). Los mencionados planes se encuentran enfocados hacia la implementación de acciones concretas que se alinean a los cinco ejes estratégicos previstos por la AGA, que son: el mejoramiento de servicios públicos; el incremento de la integridad pública; la gestión efectiva y eficiente de los recursos públicos; la creación de comunidades más seguras; y el incremento de la responsabilidad corporativa y rendición de cuentas institucional y del sector privado.

Si bien el enfoque en temas de gobierno abierto se ha dado principalmente en las esferas nacionales y federales, han ido surgiendo experiencias a nivel local en varios países de la región latinoamericana como así también se han ido estableciendo mecanismos de control y seguimiento de las administraciones públicas, fundamentalmente a través de la elaboración de distintos índices.

En tal sentido se puede mencionar el caso de Chile que desde el 2008 cuenta con las leyes 20.285 y la 20.500, sobre acceso a la información pública y sobre asociaciones y participación ciudadana, respectivamente. En dicho marco se ha creado el Consejo para la Transparencia (CPLT), el cual establece un mecanismo constante de control y seguimiento de las administraciones públicas y, desde el año 2012 mide el nivel de cumplimiento de las municipalidades en términos de publicidad y promoción de la información pública a través del Índice de Transparencia Activa Municipal (ITAM). Asimismo, en el 2016 se presentó la propuesta de la creación del Índice Institucional para el Gobierno Abierto Municipal (IIGAM) que permite observar y medir qué tanto se cumplen las disposiciones referidas a gobierno abierto en las administraciones locales chilenas (Bonivento, 2016).

8. Open Goverment Partnership (OGP) o Alianza para el Gobierno Abierto consiste en una organización multilateral fundada en Septiembre de 2011. Está compuesta por un grupo de 8 paises (Brasil, Estados Unidos, Filipinas, Indonesia, México, Noruega, Reino Unido y Sudáfrica). El OGP está vinculado hasta el momento con más de 70 paises. Los gobiernos participantes de la Alianza se comprometen individualmente a crear su Plan de Acción con la Sociedad Civil de forma colectiva. El objetivo fundamental de la OGP es promover una cultura de Gobierno Abierto para empoderar a la ciudadanía, generar resultados y difundir los ideales de la apertura y la participación del Siglo XXI. Los desafíos del OGP se basan en los principios de transparencia, compromiso y participación ciudadana, y tecnología e innovación. 
Asimismo, Brasil es uno de los dos países latinoamericanos que forman parte del grupo de miembros fundadores de la Alianza por el Gobierno Abierto (AGA) y se destaca por sus importantes avances en la materia. Particularmente, con el objetivo de medir la calidad de la transparencia de los gobiernos y en base a las exigencias contenidas en las leyes $N^{\circ} 101 / 2000$ (de Responsabilidad Fiscal- LRF), N¹31/2009 (de Transparencia) y la $\mathrm{N}^{\circ}$ 12.527/2011 (de Acceso a la Información. LAI), se han creado distintos indicadores para comprobar si las obligaciones se cumplen o no. Así, se destacan las metodologías de la Escala Brasil Transparente (EBT) creada en el año 2015 que mide la transparencia publica de estados y municipios sobre la base de las provisiones de LAI, el Índice de Ciudad Transparente desarrollado en 2014 para evaluar la transparencia de la gestión pública municipal de las ciudades sede de la Copa Mundial de Futbol del mismo año, y el Índice de Transparencia que desde el 2010 evalúa los portales de transparencia de los municipios con más de 200 mil electores (Miola, et.al. 2018). Adicionalmente, resulta relevante el modelo planteado por un grupo de investigadores denominado Índice de Transparencia de la Gestión Pública Municipal (ITGP-M) que apunta a analizar la relación entre niveles de transparencia y de desarrollo socioeconómico de los municipios del país (Ferreira Cruz, et. al. 2012).

Por su parte, Uruguay cuenta con un importante avance en cuanto a los procesos de gestión del acceso a la información pública, y se destaca por la implementación del Índice de Transparencia Activa en Línea (ITAEL) que desde el año 2013 mide el nivel de transparencia del gobierno en sus distintas escalas y el cumplimiento con la normativa vigente por parte de todos los sujetos obligados por la Ley de Acceso a la Información Publica N¹8.381 y su decreto reglamentario 232/10.(Ponce, 2017; CAinfo y UCU 2017).

En tanto que Colombia y México también cuentan con metodologías en dicho sentido, destacándose en el primero el Índice de Transparencia de las Entidades Públicas Municipales (ITEP-M), el cual busca medir los riesgos de corrupción, es decir, mide las condiciones institucionales de los municipios que puedan favorecer hechos de corrupción en la gestión administrativa, para prevenir que los mismos ocurran (Corporación Transparencia por Colombia, 2017). En México se destaca el Índice de Transparencia Municipal Básico (ITMb), que busca reflejar el grado cuantitativo de transparencia con que los gobiernos municipales desarrollan su gestión (Torres, 2012). Por último, en Argentina, la Ley de Acceso a la Información Pública sancionada el 15 de Septiembre de 2016 puso en evidencia la necesidad de tener mediciones comparadas de transparencia. Se han realizado previamente índices de transparencia activa, particularmente dos: el Índice de datos abier- 
tos de ciudades de Argentina (IDACA) de Open Knowledge International y el Índice de transparencia presupuestaria provincial (ITPP) del Centro de implementación para las políticas públicas para la equidad y el crecimiento (CIPPEC). Mientras el IDACA analiza a todos los municipios que en el año 2016 tenían portal de datos, el ITPP estudia la información presupuestaria de cada provincia.

Por su parte, el IDACA analiza el nivel de apertura de datos de los municipios y se focaliza en la legibilidad, accesibilidad y calidad de la información y del motor de búsqueda. Es decir, el objetivo central del índice reside en verificar si los datos que se publican cumplen con un formato digital abierto y reutilizable, más que verificar si el gobierno municipal publica y en qué grado ciertos parámetros de transparencia activa. Por ello toma algunas áreas de política pública (no todas), y se fija si los datos que se publican en esas áreas cumplen con los estándares de apertura y reutilización requeridos desde un punto de vista ideal. Las áreas son: el medio ambiente, los resultados electorales, el presupuesto, el transporte público, los lugares públicos, y las compras y licitaciones. En este esquema, el objetivo principal es que los indicadores sean de fácil acceso para las personas, por ello los datos relevados corresponden a las cuestiones básicas que diariamente preocupan a los ciudadanos.

Por otro lado, el ITPP evalúa de manera detallada la información presupuestaria pasada, presente y proyectada de las provincias argentinas. Los ejes centrales de este enfoque son: las leyes presupuestarias, el presupuesto plurianual, el presupuesto ciudadano, los informes de ejecución de ingresos y gastos, las transferencias de la Nación, las transferencias a los municipios y la recaudación tributaria. A diferencia del IDACA, el ITPP estudia de manera exhaustiva los datos presupuestarios de cada lugar.

En definitiva, ambos índices son complementarios, aunque aplicados en distintos niveles (municipal el índice datos abiertos, y provincial el índice del CIPPEC) y constituyen un gran avance en el estudio de la transparencia. El IDACA provee un enfoque de cómo se muestran los datos municipales en función de las necesidades del ciudadano. En cambio, el ITPP aborda la cuestión presupuestaria de manera pormenorizada para un conocimiento técnico del nivel de transparencia de cada provincia.

Por último, es posible hallar otros dos índices en Argentina que sólo han estado vigentes durante un año. Por un lado, el índice de Páginas web municipales realizado por CIPPEC (Centro de Implementación de Políticas Públicas para la Equidad y el Crecimiento) según el Programa de Desa- 
rrollo Local, junto con la Universidad de San Andrés bajo el Programa de Gobierno Electrónico. Este índice publicado en 2014 tuvo por finalidad medir, en forma regular y comparada, el nivel de desarrollo de los portales municipales. Se centró en el concepto de gobierno electrónico, al analizar los contenidos y usabilidad de los portales. Con este fin, analizó la web de 119 municipios de Argentina cuya población supera los 50.000 habitantes, además de las capitales provinciales. Según CIPPEC, se observó una brecha entre el uso y el desarrollo de TIC entre municipios grandes y medianos (mayores y menores a 200.000 habitantes).

Por otro lado, el índice de "Visibilidad Fiscal en Municipios Argentinos (IVF)" es publicado por el Instituto Argentino de Análisis Fiscal (IARAF) en el año 2017. Se calcula sobre una muestra de 100 municipios argentinos a partir de la exploración de la información de carácter fiscal publicada en los sitios web oficiales de gobierno. Según el índice, en promedio, se difunde poco menos de la mitad de la información fiscal necesaria para el control de los gobiernos locales.

\section{MET O D O L O G ÍA}

Con el objetivo de poder cuantificar el nivel de transparencia a nivel municipal, se construye un índice de transparencia municipal. El mismo se calcula considerando el aporte teórico de Darbishire (2010) y teniendo en cuenta las prácticas de transparencia de los gobiernos locales de la región. Este índice se aplica sobre una muestra de 25 ciudades correspondientes a las ciudades capitales de provincia de Argentina incluidas el municipio de Bahía Blanca, lugar de residencia de los autores y CABA. Se eligió analizar las ciudades capitales de provincia para obtener una mayor representatividad del estudio en cuanto a población se refiere.

Se exploran los sitios web municipales para determinar la presencia o no de determinados elementos de transparencia, fundamentalmente pasiva. Los datos fueron relevados entre Diciembre de 2017 y Marzo de 2018. Se seleccionaron 20 indicadores con datos objetivos sobre los siguientes aspectos: presupuesto, remuneraciones, subsidios, contratos públicos, declaraciones juradas, acceso a la información, reuniones abiertas y controles institucionales. Dada la gran diversidad de índices existentes, nos decidimos por tomar como punto de partida los criterios ideales de transparencia activa exhibidos en Darbshire (2010), y seleccionar los referidos al gasto público (que incluye subsidios, remuneraciones, asignaciones presupuestarias), a las declaraciones juradas, al presupuesto, y a las contrataciones. En materia 
de transparencia pasiva, nos decidimos por verificar la existencia de un sistema en línea centralizado, así como del tipo de respuestas que se publican, si son de acceso público o no. Es decir, medimos el derecho de acceso a la información sólo en su arquitectura estructural, pero no evaluamos si las respuestas son veraces, oportunas y completas. Los indicadores poseen tres puntuaciones posibles: 0 (el elemento está ausente), 0,5 (se encuentra incompleto) o 1 (está presente), siguiendo la metodología de otros índices como el índice de Visibilidad Fiscal.

En una primera instancia, se asigna un peso o ponderador a cada una de las variables relevadas, según la importancia relativa que se considera deben tener en el índice general. De esta manera, a cada variable i en cada localidad j le corresponde el valor dij. A su vez, se denomina a los ponderadores como pi. Evidentemente que para cada variable, el ponderador es el mismo para todos los municipios. Por último, se construye el Índice de Transparencia Municipal para cada municipio j:

Ij $=\Sigma$ pi.dij $x 100$

Siendo:

pi = ponderador asignado a cada ítem i.

dij = puntaje obtenido en la difusión de cada ítem i en cada municipio j.

$\mathrm{i}=$ ítems de información relevada, que van de 1,2 ..25.

j= muestra de 25 municipios de todo el país.

Posteriormente, se ha considerado que cada uno de los aspectos tiene un peso relativo equivalente a los fines de determinar la transparencia municipal. Luego, cada uno de los indicadores recibe el mismo valor.

\section{RES ULT A D O S}

El índice de Transparencia Municipal de Argentina (ITMA) ofrece una métrica que permite detectar cuáles son los municipios más y menos transparentes en Argentina. A su vez, es posible establecer ciertas relaciones preliminares entre el índice obtenido y algunas características socio-demográficas de los municipios tales como el tamaño y el nivel de ingresos del municipio. 


\section{ÍNDICE DE TRANSPARENCIA MUNICIPAL DE ARGENTINA PONDERADO}

El índice de transparencia elaborado para cada municipio puede variar entre 0 y 100, siendo más transparente un gobierno cuanto más cercano a cien se encuentre su valor. Los ponderadores asignados a cada variable se exponen a continuación (Tabla 1).

TABLA 1:

VARIABLES INCLUIDAS EN EL ÍNDICE DE TRANSPARENCIA

\begin{tabular}{|c|c|c|}
\hline DIMENSIÓN & INDICADOR & PONDERADOR \\
\hline Presupuesto & $\begin{array}{l}\text { Publicación de las ordenanzas presupuestarias } \\
\text { Publicación de la normativa fiscal (ordenanza tributaria) } \\
\text { Informe de ejecución anual de Presupuesto }\end{array}$ & $\begin{array}{l}0.1 \\
0.2 \\
0.1\end{array}$ \\
\hline Remuneraciones & $\begin{array}{l}\text { Información sobre las remuneraciones de empleados y } \\
\text { funcionarios (por cargo o persona) }\end{array}$ & 0.03 \\
\hline Subsidios & $\begin{array}{l}\text { Información básica (tipo, monto, duración, intermediarios) } \\
\text { Nómina o listado de beneficiarios }\end{array}$ & $\begin{array}{l}0.02 \\
0.02\end{array}$ \\
\hline Declaraciones juradas & $\begin{array}{l}\text { Declaración jurada anual de Intendente } \\
\text { Declaración jurada anual de Secretarios }\end{array}$ & $\begin{array}{l}0.1 \\
0.1\end{array}$ \\
\hline Contratos públicos & $\begin{array}{l}\text { Descripción de contratos públicos vigentes } \\
\text { Descripción de llamados a contrataciones (licitaciones) } \\
\text { Publicación de los pliegos de bases y condiciones }\end{array}$ & $\begin{array}{l}0.05 \\
0.05 \\
0.05\end{array}$ \\
\hline Reuniones abiertas & La agenda del intendente, ¿es pública? & 0.05 \\
\hline $\begin{array}{l}\text { Acceso a la } \\
\text { información }\end{array}$ & $\begin{array}{l}\text { Descripción del procedimiento para solicitar información } \\
\text { Existencia de un mecanismo online trazable para el ciudadano } \\
\text { para solicitar información } \\
\text { Publicación online de las respuestas a la información } \\
\text { demandada y si son de acceso público }\end{array}$ & $\begin{array}{l}0.05 \\
0.05 \\
0.05\end{array}$ \\
\hline
\end{tabular}

Estos ponderadores fueron establecidos ad-hoc teniendo en cuenta por un lado, los pesos asignados por otros índices previamente descriptos en la revisión, y por el otro, bajo el criterio de otorgar mayor importancia relativa a ítems no susceptibles de transparencia en la práctica. Luego, de acuerdo a la versión ponderada del índice, los grupos de información con mayor 
3 | Construcción de un indice para medir la transparencia ... F. Ciucci, L. Díaz, M. Alderete y S. Linares. importancia relativa son información sobre presupuesto y declaraciones juradas.

TABLA 2:

ESTADÍSTICOS DESCRIPTIVOS

\begin{tabular}{|llllll|}
\hline & N & Mínimo & Máximo & Media & Desv. típ. \\
\hline ITMA & 25 &, 00 &, 85 &, 3040 &, 23376 \\
\hline
\end{tabular}

FUENTE: ELABORACIÓN PROPIA.

Se observa que en promedio el Índice de Transparencia Municipal asume un valor de 0,30 para los 25 municipios analizados. Este valor refleja que tan sólo el $30 \%$ de la información sobre transparencia, considerada relevante en este estudio, es publicada en los sitios web de los municipios, existiendo divergencias entre municipios. A continuación en la Tabla 3, se presenta el ranking obtenido de los municipios. Mientras que Córdoba alcanza el puntaje más alto de 0.85, seguido de Ciudad de Buenos Aires, Bahía Blanca y Ushuaia con el segundo, tercer y cuarto lugar respectivamente, Formosa, Posadas y San Luis son los municipios con peor posicionamiento relativo.

TABLA 3:

ÍNDICE DE TRANSPARENCIA MUNICIPAL DE ARGENTINA

\begin{tabular}{|lll|lll|}
\hline RANKING & MUNICIPIO & \multicolumn{1}{l|}{ ITMA } & RANKING & MUNICIPIO & ITMA \\
\hline 1 & Córdoba & 0.85 & 14 & Paraná & 0.26 \\
2 & CABA & 0.78 & 15 & La Plata & 0.25 \\
3 & Bahía Blanca & 0.625 & 16 & Neuquén & 0.25 \\
4 & Ushuaia & 0.58 & 17 & Santiago del Estero & 0.25 \\
5 & La Rioja & 0.5 & 18 & Corrientes & 0.16 \\
6 & San Juan & 0.485 & 19 & Río Gallegos & 0.15 \\
7 & Catamarca & 0.4 & 20 & Rawson & 0.11 \\
8 & Santa Fe & 0.4 & 21 & Salta & 0.05 \\
9 & Mendoza & 0.35 & 22 & Jujuy & 0.025 \\
10 & Santa Rosa & 0.3 & 23 & Formosa & 0 \\
11 & Tucuman & 0.3 & 24 & Posadas & 0 \\
12 & Viedma & 0.265 & 25 & San Luis & 0 \\
13 & Resistencia & 0.26 & & & \\
\hline
\end{tabular}


Este ranking concuerda en cierta medida con los índices de nivel municipal mencionados previamente, ya que los municipios que ocupan los primeros puestos son los mismos, tales como CABA, Bahía Blanca y Córdoba. Por otro lado, para comprobar si el índice es robusto, se decidió cambiar los ponderadores de las variables de forma tal que cada uno refleje la inversa del desvío estándar de la variable o indicador. De esta manera, se obtienen los siguientes resultados.

TABLA 4:

ÍNDICE DE TRANSPARENCIA MUNICIPAL DE ARGENTINA 2

\begin{tabular}{|lll|lll|}
\hline RANKING & MUNICIPIO & \multicolumn{1}{l}{ ITMA2 } & RANKING & MUNICIPIO & ITMA2 \\
\hline 1 & Córdoba & 26.32963 & 14 & Resistencia & 6.75491814 \\
2 & CABA & 21.7166454 & 15 & La Plata & 6.58922318 \\
3 & Bahía Blanca & 20.5616666 & 16 & Neuquén & 6.58922318 \\
4 & Ushuaia & 16.0183702 & 17 & Santiago del Estero & 6.58922318 \\
5 & La Rioja & 14.0141578 & 18 & Paraná & 4.6388813 \\
6 & Viedma & 12.0596218 & 19 & Corrientes & 4.3661825 \\
7 & Catamarca & 11.7047568 & 20 & Rawson & 2.25014565 \\
8 & San Juan & 11.1886135 & 21 & Jujuy & 1.05801842 \\
9 & Santa Fe & 11.0783789 & 22 & Salta & 0.05 \\
10 & Santa Rosa & 9.60433662 & 23 & Formosa & 0 \\
11 & Mendoza & 9.51581817 & 24 & Posadas & 0 \\
12 & Tucumán & 8.70526003 & 25 & San Luis & 0 \\
13 & Río Gallegos & 7.05154691 & & & \\
\hline
\end{tabular}

FUENTE: ELABORACIÓN PROPIA.

Como es posible observar, el cambio de ponderadores no generó diferencias sustanciales con el ponderador ad-hoc utilizado inicialmente. Incluso las ciudades que ocupan los primeros 5 puestos son las mismas.

\section{ÍNDICE DE TRANSPARENCIA MUNICIPAL DE ARGENTINA SIN PONDERAR}

Por otro lado, para comprobar si el índice es robusto, se decidió eliminar los ponderadores de las variables (Tabla 5). Este cambio no generó diferencias sustanciales ya que las ciudades que ocupan los primeros 5 puestos son las mismas; entre ellos Bahía Blanca y Córdoba. 
TABLA 5:

RESULTADOS GENERALES

\begin{tabular}{|c|c|c|c|c|c|}
\hline \multirow{2}{*}{$\begin{array}{c}\text { RANKING } \\
1\end{array}$} & \multirow{2}{*}{$\begin{array}{l}\text { MUNICIPIO } \\
\text { Córdoba }\end{array}$} & \multirow{2}{*}{$\begin{array}{c}\text { ITMA sin ponderar } \\
71\end{array}$} & \multirow{2}{*}{$\begin{array}{l}\text { RANKING } \\
14\end{array}$} & \multicolumn{2}{|c|}{ MUNICIPIO ITMA sin ponderar } \\
\hline & & & & Neuquén & 21,42 \\
\hline 2 & CABA & 64,28 & 15 & Resistencia & 21,42 \\
\hline 3 & Bahía Blanca & 60,71 & 16 & Río Gallegos & 21,42 \\
\hline 4 & Ushuaia & 50 & 17 & Santiago del Estero & 21,42 \\
\hline 5 & La Rioja & 42,85 & 18 & Corrientes & 14,28 \\
\hline 6 & Catamarca & 35,71 & 19 & Paraná & 14,28 \\
\hline 7 & San Juan & 35,71 & 20 & Rawson & 7,14 \\
\hline 8 & Santa Fe & 35,71 & 21 & Jujuy & 3,57 \\
\hline 9 & Viedma & 32,14 & 22 & Salta & 3,57 \\
\hline 10 & Mendoza & 28,57 & 23 & Formosa & 0 \\
\hline 11 & Santa Rosa & 28,57 & 24 & Posadas & 0 \\
\hline 12 & Tucumán & 28,57 & 25 & San Luis & 0 \\
\hline 13 & La Plata & 21,42 & & & \\
\hline
\end{tabular}

FUENTE: ELABORACIÓN PROPIA.

Como se observa en la Tabla 5, los municipios que ocupan los cuatro primeros puestos en el ITMA construido son Córdoba, Ciudad de Buenos Aires, Bahía Blanca y Ushuaia. Estos municipios son los que disponen de mayor cantidad de información sobre los indicadores seleccionados. A su vez, de acuerdo a la media del ITMA (Tabla 6) son municipios que se encuentran muy por encima del valor promedio del ITMA. En particular, 12 de los 25 municipios analizados se encuentran por encima del ITMA medio.

A su vez, si comparamos el ITMA ponderado con el ITMA sin ponderar, se observa que los municipios de Viedma y Río Gallegos ganan posiciones en el ranking, ante el mayor peso otorgado a las declaraciones juradas y presupuesto, mientras que los municipios de Paraná y Resistencia empeoran.

TABLA 6:

ESTADÍSTICOS DESCRIPTIVOS

\begin{tabular}{|llllll|}
\hline & N & Mínimo & Máximo & Media & Desviación estándar \\
\hline ITMA & 25 &, 00 & 71,00 & 26,5504 & 20,05967 \\
\hline
\end{tabular}

FUENTE: ELABORACIÓN PROPIA 


\section{COMPARACIÓN DEL ITM CON LOS ÍNDICES EXISTENTES} EN ARGENTINA

El ITMA ponderado es posible compararlo con los índices existentes en la Argentina referidos al tema: el índice de datos Abiertos de las Ciudades de Argentina; el índice de Visibilidad Fiscal en Municipios de Argentina; el índice de páginas web municipales y el Índice de Transparencia Presupuestaria Provincial, estos dos últimas publicados por CIPPEC. Como se puede observar en la Tabla 7, al año 2018 tan sólo dos índices estaban en vigencia.

El ITMA incluye entre sus dimensiones a presupuesto, contratos públicos y remuneraciones, de la misma manera que IDACA y VFMA. Por otro lado, incorpora la publicación de las declaraciones juradas (DDJJ) de igual forma que el índice de páginas web municipales, aunque éste último ya no se encuentra en vigencia. Sin embargo, la visibilidad fiscal no necesariamente implica transparencia. En este sentido, el ITM introduce aspectos de transparencia activa que no han sido considerados por el resto tales como información sobre subsidios y agenda abierta del intendente, y a su vez, incluye nociones de transparencia pasiva, como el derecho de solicitar información pública por parte de los ciudadanos, que exista información publicada al respecto así como un sistema online para recibir las solicitudes de información. 
TABLA 7: CUADRO COMPARATIVO DEL ITM

\begin{tabular}{|c|c|c|c|c|c|}
\hline & ITMA & $\begin{array}{c}\text { IDACA/ } \\
\text { Open data index }\end{array}$ & VFMA & $\begin{array}{l}\text { Índice de páginas } \\
\text { web municipales }\end{array}$ & ITPP \\
\hline Vigencia & 2018 & $2016-2018$ & 2017 & 2014 & 2013-2018 \\
\hline $\begin{array}{l}\text { Municipios } \\
\text { relevados }\end{array}$ & $\begin{array}{l}23 \text { municipios } \\
\text { capitales de } \\
\text { provincia, junto } \\
\text { con CABA y Bahía } \\
\text { Blanca }\end{array}$ & $\begin{array}{l}24 \text { municipios } \\
\text { versión } 2017\end{array}$ & 100 municipios & $\begin{array}{l}119 \text { municipios } \\
\text { de }>50 \text { mil hab. }\end{array}$ & $\begin{array}{l}24 \text { provincias } \\
\text { argentinas }\end{array}$ \\
\hline Escala & 0 a 1 & 0 a 1 & 0 a 1 & 0 a 1 & 0 a 10 \\
\hline $\begin{array}{l}\text { Método de } \\
\text { recolección } \\
\text { de datos }\end{array}$ & $\begin{array}{l}\text { Exploración de } \\
\text { los portales web } \\
\text { municipales; } 1 \text { : si } \\
\text { existe, 0,5: existe } \\
\text { en forma parcial; } \\
\text { 0: no existe }\end{array}$ & $\begin{array}{l}\text { ¿Cuál es el estado } \\
\text { de apertura de } \\
\text { datos en los } \\
\text { municipios de } \\
\text { Argentina?" }\end{array}$ & $\begin{array}{l}\text { 1: si existe, 0,5: } \\
\text { existe en forma } \\
\text { parcial; 0: no } \\
\text { existe } \\
\text { presupuesto } \\
\text { (informe } \\
\text { ejecución, }\end{array}$ & $\begin{array}{l}\text { Exploración } \\
\text { de los portales } \\
\text { web locales, } \\
\text { contenidos y } \\
\text { usabilidad de las } \\
\text { páginas web, 1: } \\
\text { si existe; 0: no }\end{array}$ & $\begin{array}{l}\text { Exploración de } \\
\text { los portales web } \\
\text { provinciales } \\
\text { presupuesto } \\
\text { (normas, informe } \\
\text { ejecución, } \\
\text { recursos, }\end{array}$ \\
\hline $\begin{array}{l}\text { Dimensiones } \\
\text { incluidas }\end{array}$ & $\begin{array}{l}\text { presupuesto } \\
\text { (ordenanza, } \\
\text { normas, informe } \\
\text { ejecución) } \\
\text { contratos } \\
\text { públicos } \\
\text { remuneraciones } \\
\text { DDJJ } \\
\text { subsidios } \\
\text { reuniones } \\
\text { abiertas (agenda) } \\
\text { derecho de } \\
\text { acceso a la } \\
\text { información } \\
\text { (información } \\
\text { sobre el derecho, } \\
\text { sistema } \\
\text { habilitado en el } \\
\text { portal, etc) }\end{array}$ & $\begin{array}{l}\text { gasto público } \\
\text { transporte } \\
\text { público } \\
\text { resultados } \\
\text { electorales } \\
\text { compras y } \\
\text { contrataciones } \\
\text { lugares públicos } \\
\text { ordenanzas } \\
\text { límites } \\
\text { administrativos } \\
\text { medio ambiente } \\
\text { sueldos } \\
\text { funcionarios }\end{array}$ & $\begin{array}{l}\text { normativa } \\
\text { tributaria; deuda } \\
\text { pública) } \\
\text { licitaciones } \\
\text { públicas } \\
\text { planta personal }\end{array}$ & $\begin{array}{l}\text { existe } \\
\text { presupuesto } \\
\text { DDJJ }\end{array}$ & $\begin{array}{l}\text { divulgación); } \\
\text { presupuesto } \\
\text { ciudadanos }\end{array}$ \\
\hline $\begin{array}{l}\text { Items mas } \\
\text { ponderados }\end{array}$ & $\begin{array}{l}\text { Presupuesto, } \\
\text { contrataciones } \\
\text { públicas y DDJJ }\end{array}$ & $\begin{array}{l}\text { Si los datos } \\
\text { son en formato } \\
\text { abierto, datos en } \\
\text { dominio público } \\
\text { (para todo item) }\end{array}$ & $\begin{array}{l}\text { Presupuesto } \\
\text { y ejecución } \\
\text { presupuestaria }\end{array}$ & $\begin{array}{l}\text { Presupuesto, } \\
\text { DDJJ }\end{array}$ & $\begin{array}{l}\text { Presupuesto } \\
\text { (informe de gasto } \\
\text { y ejecución) leyes } \\
\text { de presupuesto y } \\
\text { deuda pública }\end{array}$ \\
\hline $\begin{array}{l}\text { Mejor municipio } \\
\text { reciente }\end{array}$ & Córdoba, BA.BCA & Córdoba, CABA & CABA, Córdoba & $\begin{array}{l}\text { Moron y Gral } \\
\text { Pueyrredón }\end{array}$ & $\begin{array}{l}\text { Provincia de } \\
\text { Buenos Aires, } \\
\text { CABA }\end{array}$ \\
\hline $\begin{array}{l}\text { Valor promedio } \\
\text { reciente }\end{array}$ & 0,27 & 0,41 & 0.42 & 0,36 & 7,8 \\
\hline Fuente & & $\begin{array}{l}\text { Fundación } \\
\text { Conocimiento } \\
\text { Abierto, desde } \\
2016 \text { https:// } \\
\text { index.okfn.org/ } \\
\text { methodology/ }\end{array}$ & $\begin{array}{l}\text { Instituto } \\
\text { Argentino } \\
\text { de Analisis } \\
\text { Fiscal IARAF } \\
\text { http://www. } \\
\text { agaargentina.org/ } \\
\text { category/ogp/ }\end{array}$ & $\begin{array}{l}\text { CIPPEC www. } \\
\text { cippec.org }\end{array}$ & $\begin{array}{l}\text { CIPPEC www. } \\
\text { cippec.org }\end{array}$ \\
\hline
\end{tabular}


TABLA 8:

\section{POBLACIÓN SEGÚN TIPO DE MUNICIPIO}

\begin{tabular}{|lccccc|}
\hline Municipios & N & Media & Desviación estándar & Mínimo & Máximo \\
\hline ITMA < media & 13 & 281185,8462 & 166472,00592 & 81787,00 & 699523,00 \\
ITMA $>$ media & 12 & 548378,6667 & 837085,75450 & 46767,00 & $2,89 \mathrm{E}+06$ \\
Total & 25 & 409438,4000 & 594624,51072 & 46767,00 & $2,89 \mathrm{E}+06$ \\
\hline
\end{tabular}

FUENTE: ELABORACIÓN PROPIA

Por otro lado, tras calcular la correlación bivariada entre tamaño del municipio y el indicador de transparencia obtenido, se encuentra que existe una correlación estadísticamente significativa al 5 y positiva. Es decir, municipios de mayor tamaño se corresponden con municipios con mayores niveles de transparencia. Luego, el nivel de transparencia del municipio estaría relacionado con el tamaño del municipio.

Por último, dado que el tamaño del municipio no es una variable representativa del nivel de ingresos del municipio, se estableció el mismo tipo de análisis con la variable recaudación tributaria/ingresos corrientes. Cabe aclarar que estos datos corresponden a la provincia, ya que la información disponible a nivel municipal se encuentra sólo en algunos casos publicada en la sección presupuesto de los portales web municipales. Los datos utilizados a nivel provincial provienen de la Fundación Libertad, del informe sobre el Índice de Desempeño Provincial 2018.

TABLA 9:

COMPARACIÓN DEL ITM MEDIO SEGÚN NIVEL DE INGRESOS DE LA PROVINCIA

\begin{tabular}{|lccr|}
\hline Recaudación tributaria/ingresos corrientes & Media de ITMA & N & Desv. típ. \\
\hline inferior a 30\% & 20,1089 & 19 & 15,25441 \\
superior a 30\% & 44,1960 & 5 & 22,11759 \\
Total & 25,1271 & 24 & 19,15823 \\
\hline
\end{tabular}

Como se puede observar de la Tabla 9, los municipios cuyas provincias poseen una recaudación tributaria respecto de los ingresos corrientes superior al 30\% alcanzan un índice de transparencia municipal de 44.20 que 
duplica el obtenido por el resto de los municipios. Esta diferencia es estadísticamente significativa según la Tabla de ANOVA para un nivel de significancia del 1\%. Por lo tanto, se deduce que hay relación entre los ingresos del municipio y los niveles de transparencia.

\section{O N C L U S I O N E S E}

\section{M P L I CANCIASPRÁCTICA S}

El principal aporte de este trabajo consiste en brindar una medida del nivel de transparencia de los gobiernos municipales. En particular, se introduce no solo la transparencia activa sino también la noción de transparencia pasiva, ya que los escasos índices de nivel municipal existentes en Argentina se centraban únicamente en las nociones de datos abiertos y de transparencia presupuestaria. Por el contrario, el Índice de Transparencia Municipal (ITM) propuesto es más amplio que aquellos al requerir información sobre contrataciones públicas y declaraciones juradas de los funcionarios. Al respecto, los municipios más transparentes en Argentina -de la muestra seleccionada- son Córdoba, CABA, Bahía Blanca y Ushuaia. Este orden de transparencia se mantiene independientemente de la metodología empleada para calcular el índice (sea con o sin ponderadores). El nivel de transparencia promedio en Argentina es de 0.30, y los municipios mejor posicionados se encuentran muy por encima de este valor. Por otra parte, se observa cierta relación entre el tamaño de los municipios y el nivel de transparencia obtenido.

Por otro lado, la metodología aplicada para la confección del índice puede ser replicada en otros países de la región dado que los indicadores utilizados son objetivos y detectables a partir de los sitios web oficiales. El índice permite no sólo la comparación entre municipios en términos de transparencia, sino que brinda una herramienta de política para mejorar la gestión de los gobiernos municipales así como proveer de información al sector privado con fines productivos.

Entre las limitaciones del trabajo, los datos del índice corresponden al año 2018. Actualizar la información a partir de la exploración de los portales de datos abiertos o sitios web es una tarea ardua. Por ese motivo, sería interesante crear un algoritmo para recopilar la información necesaria más eficientemente. Por otro lado, la versión del índice ponderado utiliza ponderadores establecidos ad-hoc basados en la evidencia empírica e interpretación individual. Por último, dado que el número de capitales de provincia en Argentina es reducido, no es posible con esta muestra realizar estudios 
de regresión que capturen la interrelación entre las variables analizadas. A futuro, se espera ampliar el número de municipios analizados para permitir este análisis. 


\section{R E F E R Ê N C I A S}

Alderete, M.V. y Linares, S. (2017). Participación ciudadana: el caso de la ordenanza de Acceso a la Información Pública de la Municipalidad de Bahía Blanca. LII Reunión Anual de la Asociación Argentina de Economía Política. Bariloche (15-17 Noviembre de 2017).

Angelidou, M. (2014). Smart city policies: A spatial approach. Cities 4, 3-11.

Bonivento Hernández, J. (2016). Diseño institucional para el gobierno abierto municipal: propuesta de medición y análisis del caso chileno. Documentos y Aportes en Administración Pública y Gestión Estatal, 16 (27), 101-128.

Centro de Archivos y Acceso a la Información Pública (CAinfo) \& Universidad Católica del Uruguay (UCU). Índice de Transparencia Activa en Línea. Relevamiento 2017. Disponible en: https://ucu.edu.uy/sites/default/files/pdf/2018/itael-2017-indice-transparencia-activa-linea.pdf

Centro de implementación para las políticas públicas para la equidad y el crecimiento. (2017). Índice de transparencia presupuestaria provincial. Disponible en: https:// www.cippec.org/.

Coase, R. (1937). The nature of the firm. Económica, 4 (16), 386-405.

Corporación Transparencia por Colombia (2017). Índice de Transparencia Municipal.

Resultados 2015-Abril 2016. Colección de Documentos Observatorio de Integridad $\mathrm{N}^{\circ}$ 17. Bogotá, Colombia. Disponible en: https://masinformacionmasderechos.co/ wp-content/uploads/2017/05/17.-Indice-de-Transparencia-Municial-2015-2016.pdf

Cuillier, D., y Piotrowski, S. J. (2009). Internet information-seeking and its relation to support for access to government records. Government Information Quarterly, 26 (1), 441-449.

Darbishire, H. (2010). Proactive transparency: the future of the right to information? A review of standards, challenges, and opportunities (English). Governance working paper series. Washington, DC: World Bank. http://documents.worldbank.org/curated/ en/100521468339595607/Proactive-transparency-the-future-of-the-right-to-information-A-review-of-standards-challenges-and-opportunities.

Davis, F. (1989). Perceived Usefulness, Perceived Ease of Use and User Acceptance of Information Technology. MIS Quarterly, 13 (3), 319-340.

Ferreira Cruz, C. et. al. (2012). Transparência da gestão pública municipal: um estudo a partir dos portais eletrônicos dos maiores municípios brasileiros. RAP- Rio de Janeiro, 46(1), 155-76.

Ferreiro, A. Dinero, política y transparencia: el imperativo democrático de combatir la corrupción, ponencia. Presentada en la Novena Conferencia Anti-Corrupción. Durban, Sudáfrica, 1999. 
Herrera Torres, H.; et al. (2017). Transparencia municipal: método de cálculo de indicador condensado. Propuesta para los gobiernos locales de Michoacán, México. Revista Enfoques, 15 (26).

Hofmann, A.; Ramírez Alujas, Á.; Bojórquez, J. (2013). La promesa del Gobierno Abierto. México: ITAIP.

Hollands, R. G. (2008). Will the real smart city please stand up?. City, 12, 303-320.

Kim, P. S., Halligan, J., Cho, N., Oh, C. H., y Eikenberry, A.M. (2005). Toward participatory and transparent governance: Report on the sixth Global Forum on Reinventing Government. Public Administration Review, 65 (6), 646-654.

Kolstad, I., y Wiig, A. (2009). Is transparency the key to reducing corruption in resource-rich countries?. World Development, 37 (1), 521-532.

Komninos, N; Pallot, M. y Schaffers, H. (2013). Special issue on smart cities and the future internet in Europe. Journal of Knowledge Economics, 4, 119-134.

Krastev, I. (2004). Shifting obsessions: three essays on the politics of anticorruption. Central European University Press.

Lascoumes, P. (1999). Corruptions. Presses de la Fondation nationale des sciences politiques.

Miola, E.; Jamil Marques, F.P.; Almada, M.P; Dias Ferreira Amorim, P.K. (2018). Avaliando Índices de Transparência. I Congreso DO INCT.DD (19 a 21 de Septiembre de 2018) Salvador- Bahía.

Montoya Morales, L.M. y Zhardez, V. (2016). Gobierno Local: construcción colectiva del conocimiento para la modernización municipal. Documento para su presentación en el VII Congreso Internacional en Gobierno, Administración y Políticas Públicas GIGAPP. (Madrid, España) del 3 al 5 de octubre de 2016.

Noveck, B. 2017. Rights-Based and Tech-Driven: Open Data, Freedom of Information, and the Future of Government Transparency, 19 Yale Hum. Rts. \& Dev. L.J. (2017). Disponible en: https://digitalcommons.law.yale.edu/yhrdlj/vol19/iss1/1

Open Knowledge International Argentina (2016). Índice de datos abiertos de ciudades de Argentina. Disponible en: https://okfn.org/network/argentina/.

Oszlak, O.; Kaufman, E. (2014). Teoría y práctica del gobierno abierto. Lecciones de la experiencia internacional. IDRC - RedGEalc - OEA.

Pereyra, S. (2013). Política y transparencia: la corrupción como problema público. Siglo Veintiuno Editores.

Ponce, M. (2017). Tan cerca y tan lejos: la transparencia en los gobiernos departamentales en Uruguay 2013-2016. Revista Uruguaya de Ciencia Politica. 26(2), 75-102. Disponible en: http://dx.doi.org/https://doi.org/10.26851/rucp.v26n2.4. 
Ramírez Alujas, A. y Dassen, N. (2014). Vientos de cambio. El avance de las políticas de gobierno abierto en América Latina y el Caribe. Banco Interamericano de Desarrollo (BID). Instituciones para el Desarrollo (IFD). División de Capacidad Institucional del Estado (ICS). Nota técnica del BID; 629

Rogers, E. M. (1995). Diffusion of Innovations. Nueva York, Free Press.

Ubaldi, B. (2013). Open Government Data: Towards Empirical Analysis of Open Government Data Initiatives. OECD Working Papers on Public Governance, No. 22, OECD Publishing, Paris.

Williamson, 0. (1981). The Economics of Organization: The Transaction Cost Approach. American Journal of Sociology, 87 (3), 548-577. 